\title{
A Cross-lag Analysis of Longitudinal Associations between Non-verbal Intelligence and Math Achievement
}

\author{
Tatiana Tikhomirova ${ }^{1,{ }^{*}, \text { Ekaterina Misozhnikova }}{ }^{1}$, Yulia Kuzmina ${ }^{2}$ and Sergey Malykh ${ }^{1}$ \\ ${ }^{1}$ Psychological Institute of Russian Academy of Education, 125009 Moscow, Russia \\ ${ }^{2}$ Natinal Research University Higher School of Economics, 2101000 Moscow, Russia
}

\begin{abstract}
The article presents the results of a longitudinal study of the relationship of nonverbal intelligence with success in teaching mathematics. A cross-lag analysis showed that at primary school age the empirical data is best described with the theoretical model of indirect influence of non-verbal intelligence. According to this model, nonverbal intelligence measured at Grade 3 of schooling affects the success in mathematics by the end of Grade 3, which, in turn, influences the success in mathematics by the end of primary school.
\end{abstract}

\section{Introduction}

Relationship between intelligence and academic success is observed in a number of cross-sectional studies carried out in different socio-economic contexts, educational conditions and ages [1-7]. These studies, including metaanalyses, reported high correlations of intelligence and academic success in various school subjects - from 0.37 to $0.63[7,8]$.

At the same time, the question of the causal relationship of intelligence and academic success requires further longitudinal studies, in which both analyzed constructs are measured in the same respondents over a certain time period [9]. For example, in a five-year longitudinal study of 70,000 British schoolchildren it was shown that intelligence measured at the age of 11 , explains almost $60 \%$ of the variability in standardized test scores in mathematics at the age of 16 [5].

At the same time, the results of other longitudinal studies indicate that the most important factor affecting the increase in the mean intelligence score with age is schooling $[10,11]$. Thus, in natural experiments involving children who are unable to study at school, it was found that these children show intellectual deficit of two standard deviations [11].

Thus, schooling might affect the mean values of nonverbal intelligence. It is possible that the differences in educational systems (both formal and conceptual) might mediate the relationship of intelligence and academic success in the course of development $[1,4,11,12]$. The purpose of this longitudinal study is to investigate the causal relationship between non-verbal intelligence and success in mathematics at primary school age in the Russian sample.

\section{Methods}

\subsection{Participants}

The sample included students of the municipal educational institution participating in a longitudinal study of academic success. The present study analyzed the data of 133 students of Grades 3 and 4.

The average age of participants at the time of the first testing was 9.82 years $(\mathrm{SD}=0.30)$, with $45.1 \%$ being boys, and $54.9 \%$ - girls.

The average age of the participants during the second testing was 10.82 years $(\mathrm{SD}=0.30)$, the number of boys was $45.1 \%$, the number of girls $-54.9 \%$.

Each participant took part in the study twice - at the end of Grades 3 and 4.

\subsection{Measures}

\subsubsection{Intelligence}

Non-verbal intelligence was measured with the 'Raven Progressive Matrices' test (RPM, [13]). It consists of 60 tasks grouped in 5 series. In series A, participants have to complement the missing part of an image. In series B they are asked to find correspondence between pairs. In series $\mathrm{C}$ the tasks are related to geometrical principles in figure changes. In series D participants have to find the structure in shuffling of figures. Series E requires the ability to analyze figures and add it to the missing parts. Each correct answer was counted as 1 score. Total scores were calculated.

\footnotetext{
Corresponding author: tikho@mail.ru
} 


\subsubsection{Mathematical achievement}

Academic success was indicated by term grades for mathematics as marked by a primary school teacher. In the statistical analysis the mean of four term grades was used.

\subsubsection{Statistical Analyses}

At the first stage the descriptive statistics were calculated for each of the analyzed variables recorded at the end of Grades 3 and 4.

At the second stage a correlation analysis of nonverbal intelligence and success in learning mathematics, as measured at the end of Grades 3 and 4, was carried out. Spearman correlation coefficients were calculated (SPSS 20.0 package).

At the third stage, a confirmatory factor analysis was conducted to develop a measurement model of nonverbal intelligence as a latent variable. This is due to the fact that one of the main conditions of latent growth modeling is factorial invariance [9]. The total scores for five series of Raven Standard Progressive Matrices were used as indicators of the latent variable, nonverbal intelligence. To compare the models of nonverbal intelligence as a latent variable obtained on the data in Grades 3 and 4, chi-square values and degrees of freedom were used (MPlus package).

At the fourth stage, cross-lagged structural equation modeling was used for the analysis of the developmental structure of causal relationships between nonverbal intelligence and success in mathematics, measured twice at primary school age. Cross-lag analysis allowed to assess: 1) autoregressive correlations reflecting the stability of each trait over time, 2) contemporaneous correlations, estimating the total variation of the variables within each of the two measurements, and 3) cross-lag correlations showing how the variation of one trait at one time point explains the variation of another trait measured in the next time point.

To compare the models, the following criteria were used: chi-square test $(p>0,05)$; root mean square error of approximation (RMSEA $\leq 0.06,90 \%$ confidence intervals - RMSEA low $=0.00$ and RMSEA high $<$ 0.08); comparative fit index (CFI $>0,95)$; Tucker-Lewis index (TLI> 0,90); Akaike Information Criterion (AIC), Bayesian information criterion (BIC) and sample-size adjusted BIC.

Five competing models of the relationship of nonverbal intelligence and success in mathematics measured at two points in time during the early school age were sequentially compared. In all structural models nonverbal intelligence was analyzed as a latent construct based on test scores for the series of Raven Progressive Matrices, and success in mathematics - as the mean of four term grades.

Model 1, autoregressive: non-verbal intelligence and success in mathematics only correlate within each measurement, none of the indicators affects the other one measured in the next time point.
Model 2, with a direct effect: non-verbal intelligence, measured in Grade 3, predicts the success in learning mathematics in Grade 4.

Model 3, with a direct effect: success in learning mathematics in third grade predicts nonverbal intelligence at Grade 4.

Model 4, reciprocal: non-verbal intelligence measured in Grade 3, predicts success in mathematics in Grade 4, and success in mathematics in Grade 3 test predicts nonverbal intelligence measured in Grade 4.

Model 5, with indirect effects: includes two indirect effects, where non-verbal intelligence measured in Grade 3 serves as a predictor, and success in learning mathematics in third grade as a mediator. The first effect involves the influence of non-verbal intelligence measured in Grade 3 on the success in learning mathematics in Grade 4 through success in mathematics in Grade 3. The second effect suggests that non-verbal intelligence in Grade 3 predicts non-verbal intelligence in Grade 4 through the success in learning mathematics in Grade 3.

\section{Results}

Table 1 presents the descriptive statistics for all indicators of non-verbal intelligence (scores for series $\mathrm{A}-\mathrm{E}$ and the total score for the test) and success in learning mathematics (mean of four term grades) in Grades 3 and 4.

Table 1. Descriptive statistics of non-verbal intelligence and success in learning mathematics in Grades 3 and 4.

\begin{tabular}{|l|c|c|}
\hline \multirow{2}{*}{ Measure } & \multicolumn{2}{|c|}{ Mean (SD) } \\
\cline { 2 - 3 } & 3rd grade & 4th grade \\
\hline Non-verbal intelligence, series A & $10,64(1,50)$ & $11,07(1,07)$ \\
\hline Non-verbal intelligence, series B & $10,22(2,16)$ & $10,62(1,64)$ \\
\hline Non-verbal intelligence, series C & $8,02(2,33)$ & $8,47(2,29)$ \\
\hline Non-verbal intelligence, series D & $8,03(2,60)$ & $8,20(2,31)$ \\
\hline Non-verbal intelligence, series E & $3,43(2,52)$ & $3,60(2,46)$ \\
\hline $\begin{array}{l}\text { Non-verbal intelligence, } \\
\text { total score }\end{array}$ & $40,63(7,86)$ & $42,02(7,13)$ \\
\hline Success in learning mathematics & $4,17(0,61)$ & $4,15(0,63)$ \\
\hline
\end{tabular}

Table 1 shows the mean values and standard deviations for the analyzed indicators. The maximum score for each series in the Standard Progressive Matrices is 12 , for the total score -60 . The minimum and maximum values for success in mathematics are 2 and 5, respectively.

According to Table 1, means of non-verbal intelligence (on the series and the entire test) increase from the third to Grade 4 with narrowing of variability of individual differences (standard deviation slightly decreases). Indicators of success in learning mathematics, on the other hand, demonstrate the stability of the means and variation range. Similar data on the age-specific ratios of cognitive development and success in learning were obtained in cross-sectional studies on the samples of Russian schoolchildren [7]. A decrease in the means from series A to E in the Raven Progressive Matrices, as well as an increase in the standard deviation, confirms the principle of progressive complexity of tasks underlying the test of nonverbal intelligence. 


\subsection{Correlation between intelligence and Math achievement at Grade 3 and Grade 4}

In the correlation analysis we studied the structure of the relationship of nonverbal intelligence and success in learning mathematics in the third and fourth grades.

Overall, statistically significant correlations - from low to high - were observed between nonverbal intelligence and success in mathematics in Grades 3 and 4. To the greatest extent success in mathematics was associated with nonverbal intelligence as indicated by the total score and the scores for D series. Thus, in Grade 3 the correlations reach 0.45 and 0.44 , respectively, and in Grade $4-0.42$ and 0.41 at $p<0.01$. Success in mathematics is weakly associated with nonverbal intelligence as indicated by the $\mathrm{C}$ series of tasks in Grade $3(\mathrm{r}=0.29, p<0.05)$ and series $\mathrm{E}$ and $\mathrm{C}$ in Grade 4 $(r=0.23$ and 0.24 , respectively, $p<0.05)$. This wide range of the correlation coefficients for success in learning mathematics with different test parameters of intelligence necessitates the analysis of non-verbal intelligence as a latent construct based on the scores on the five series of the 'Raven Progressive Matrices' test. The studies have shown that for a more adequate assessment of the relationship intelligence should be considered not as a test score, but as a latent construct, allocated on the basis of performance on a number of tests $[14,15]$.

The correlation between non-verbal intelligence scores measured twice at an interval of one academic year is $0.53, p<0.01$, and between indicators of success in mathematics reaches 0.90 at $p<0.01$, confirming the available literature on the stability of high success rates based on teachers' assessments $[14,16]$.

There is a tendency to a certain weakening of the relationship between measures of nonverbal intelligence and success in mathematics from Grade 3 to Grade 4. However, generally, the structure of the relationships remains stable.

\subsection{Cross-lag analyses of causal associations between intelligence and Math achievement}

Within the cross-lag analysis five competing models of the relationship of non-verbal intelligence and success in mathematics at primary school age were considered, first four models with direct effects, then one model with two indirect effects. Table 2 shows the fit indices for the models of the relationship between non-verbal intelligence and success in learning mathematics.

According to Table 2, among the models with direct effects a reciprocal model described the empirical data best, suggesting that intelligence in Grade 3 predicts the success in learning mathematics in Grade 4, and on the contrary, success in learning mathematics in Grade 3 predicts intelligence in Grade 4.

All autoregressive and contemporaneous relationships were statistically significant. An exception is the connection between non-verbal intelligence in Grade 3 and success in mathematics in Grade 4. Estimation of the model parameters shows that non-verbal intelligence in
Grade 3 does not make a statistically significant contribution to the variation in success in learning mathematics by the end of primary school $(\beta=0.07$, $p>0.05)$.

Table 2. Fit indices for the models of the relationship between non-verbal intelligence and success in learning mathematics.

\begin{tabular}{|c|c|c|c|c|c|c|}
\hline \multicolumn{2}{|c|}{ Indices } & M 1 & M 2 & M 3 & M 4 & M 5 \\
\hline \multirow{3}{*}{$\stackrel{\circlearrowright}{\circlearrowright}$} & AIC & 4925,6 & 4925,9 & 4921,4 & 4922,5 & 4922,5 \\
\hline & BIC & 5019,2 & 5022,3 & 5017,8 & 5021,6 & 5021,6 \\
\hline & $\begin{array}{l}\text { adj. } \\
\text { BIC }\end{array}$ & 4911,8 & 4911,7 & 4907,2 & 4907,9 & 4907,9 \\
\hline \multirow{3}{*}{$\approx$} & value & 71,5 & 69,86 & 65,31 & 64,42 & 64,42 \\
\hline & $\mathrm{df}$ & 56 & 55 & 55 & 54 & 54 \\
\hline & $p$ & 0,07 & 0,08 & 0,16 & 0,16 & 0,16 \\
\hline \multirow{2}{*}{ 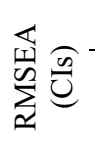 } & Estim. & 0,05 & 0,05 & 0,04 & 0,04 & 0,04 \\
\hline & $\begin{array}{c}90 \% \\
\text { CI }\end{array}$ & $\begin{array}{c}0,00- \\
0,08\end{array}$ & $\begin{array}{c}0,00- \\
0,08\end{array}$ & $\begin{array}{c}0,00- \\
0,07\end{array}$ & $\begin{array}{c}0,00- \\
0,07\end{array}$ & $\begin{array}{c}0,00- \\
0,06\end{array}$ \\
\hline \multirow{2}{*}{ 至 } & CFI & 0,97 & 0,97 & 0,97 & 0,98 & 0,99 \\
\hline & TLI & 0,97 & 0,97 & 0,97 & 0,98 & 0,98 \\
\hline
\end{tabular}

Note: AIC - Akaike Information Criterion; BIC - Bayesian information criterion; adj. BIC - sample-size adjusted BIC; $\chi^{2}$ (value, $d f, p$ ) - chi-square test (value, degrees of freedom, $p$ value); RMSEA (Estim., CIS) - root mean square error of approximation (value, $90 \%$ confidence intervals); CFI comparative fit index; TLI-Tucker-Lewis index

However, in the vast majority of research intelligence is seen as cognitive predictor of success in learning, including studies on the Russian sample [5-7]. Therefore, in the further analysis a model with indirect effects was tested where non-verbal intelligence in Grade 3 acts as a predictor, and success in learning mathematics in Grade 3 is considered as a mediator.

Estimation of the model with indirect effects indicates that autoregressive coefficients are statistically significant $(p<0.01)$ and reach values: 0.46 for non-verbal intelligence and 0.79 for the success in learning in Grades 3 and 4.

The regression coefficients of contemporaneous associations of intelligence and success in mathematics measured at each of the two time points are significantly different. Thus, in Grade 3 the relationship of non-verbal intelligence and success in mathematics is well pronounced $(\beta=0.53$ with $p<0.001)$. In Grade 4 , however, the strength of the association between these indicators significantly reduced $(\beta=0.11$ at $p<0.05)$. One of the reasons for the observed differences might be a high autoregressive factor between success in mathematics in Grades 3 and 4. According to the literature, the high temporal stability of one construct might weaken the contemporaneous association [9].

The Model 5 analyzed indirect cross-lag relationships between non-verbal intelligence and success in learning mathematics in a two-year period of primary school. According to the analysis, non-verbal intelligence in Grade 3 affects the success in learning mathematics in Grade 4 through success in mathematics in Grade 3. 
Statistically significant standardized weight of this indirect path is $0.42(p<0.001)$, after controlling for the variations related to autoregressive and contemporaneous associations.

The regression weight of the second indirect association - non-verbal intelligence in Grade 3 predicts intelligence in Grade 4 through the success in learning mathematics in Grade 3 - is significantly lower $(\beta=0.11$, $p<0.05)$.

Thus, the cross-lag analysis showed that non-verbal intelligence in Grade 3 predicts $49 \%$ of variance of success in mathematics in Grade 4 through success in mathematics in Grade 3.

\section{Discussion}

The study investigated the causal relationship between non-verbal intelligence and success in learning mathematics by means of cross-lagged structural equation modeling.

The analyzed indicators were measured twice - in Grades 3 and 4. The cross-lag analysis allowed to assess: a) the temporal stability of nonverbal intelligence and success in mathematics in the early school years, b) the total variation of the variables within each of the two measurements in Grades 3 and 4, and c) the causal relationship between non-verbal intelligence and success in mathematics in primary school.

The statistical analysis showed that among the analyzed indicators the success in learning mathematics as measured by teachers' assessments was most stable at primary school age. It is reported that teachers' marks as indicators of success in schooling are highly stable [16], which makes it difficult to analyze longitudinal relationships with other variables at each subsequent measurement. However, teacher's assessment is the only measure of success of Russian students in learning, which can be used in longitudinal studies throughout the school years - from second to eleventh grades.

For non-verbal intelligence lower autoregressive correlations were obtained in comparison with the success in mathematics, but in general they suggest the temporal stability of this psychological construct, after controlling for variation related to contemporaneous and cross-lag associations. The results correspond to the results of studies of age dynamics of intelligence (e.g., [17]). In particular, these studies reported that intelligence indicators are more stable from early adolescence to late adulthood, whereas before adolescence intelligence develops unevenly [17, 18].

The analysis of contemporaneous correlations showed that the relationship of nonverbal intelligence and success in learning mathematics differ in Grades 3 and 4. In Grade 3 strong relationships are observed between these variables, which are fully consistent with the results of cross-sectional studies involving school-age children. A strong relationship was reported between intelligence and academic success - from 0.37 to $0.63[5,7,8]$. In Grade 4 a much weaker correlation was obtained, which, as was indicated above, is due to the high autoregressive coefficients obtained for the success in learning mathematics in Grades 3 and 4.

Among the models including direct paths between non-verbal intelligence and success in mathematics, the reciprocal model assuming a bidirectional relationship of constructs over time best describes the empirical data. However, in this model the influence of non-verbal intelligence measured at an earlier time point on success in learning mathematics recorded at a later time point was not statistically significant. On the contrary, the success in learning mathematics in Grade 3 explained $21 \%$ of the variance of nonverbal intelligence in Grade $4(p<0.05)$. This result is consistent with the available literature on the significant influence of learning on the 'Raven Progressive Matrices' test performance [10, 11, 19]. However, in a longitudinal study conducted on a sample of American students [20], influence the success in learning on intelligence was not confirmed, which may be due to differences in the measurement of academic success.

The theoretical model, suggesting an indirect effect of non-verbal intelligence in Grade 3 on the success in mathematics in Grade 4 best describes the empirical data. The success in learning mathematics in Grade 3 acts as a mediator. Consequently, non-verbal intelligence in Grade 3 and success in mathematics a year after are associated through an indirect causal relationship: 'early' intelligence acts as a predictor of 'later' academic achievement in mathematics.

These results might reflect the specifics of the relationship of cognitive functioning and academic success in terms of the Russian system of education.

This study was supported by the grant from the Russian Science Foundation [grant RSF № 15-18-30055].

\section{References}

1. T.N. Tikhomirova, S.B. Malykh, M.G. Tosto, Y.V. Kovas, Psychological journal, 35, 41 (2014)

2. T.N. Tikhomirova, A.D. Modyaev, N.M. Leonova, S.B. Malykh, Psychological journal, 36, 43 (2015)

3. T. Tikhomirova, I. Voronina, J. Marakshina, E. Nikulchev, I. Ayrapetyan, T. Malykh, SHS Web of Conferences, 29, 02039 (2016)

4. S.B. Malykh, T.N. Tikhomirova, C. Zhou, W. Wei, M. Rodic, E.B. Misozhnikova, Y.A. Davydova, Y. Kovas, Voprosy psihologii, 5, 133 (2012)

5. I.J. Deary, S. Strand, P. Smith, C. Fernandez, Intelligence, 35, 13 (2007)

6. L.A. Verbitskaya, S.B. Malykh, Y.P. Zinchenko \& T.N. Tikhomirova, Psychology in Russia. State of the Art, 8, 91 (2015)

7. T.N. Tikhomirova, I.V. Voronin, E.B. Misozhnikova, S.B. Malykh, Theoretical and experimental psychology, 8, 55 (2015)

8. D. Luo, L.A. Thompson, D.K. Detterman, Intelligence, 34, 79 (2006) 
9. T.D. Little, K.J. Preacher, J.P. Selig and N.A. Card, International Journal of Behavioral Development, 31, 357 (2007)

10. C.N. Brinch, T.A. Galloway, Proceedings of the National Academy of Sciences, 109, 425 (2012)

11. R. Nisbett, J. Aronson, C. Blair, W. Dickens, J. Flynn, D. Halpern, E. Turkheimer, American Psychologist, 67, 130 (2012)

12. M. Rodic, X. Zhou, T. Tikhomirova, W. Wei, S. Malykh, V. Ismatulina, E. Sabirova, Y. Davidova, M. Tosto, J-P. Lemelin and Y. Kovas, Developmental Science, 18, 165 (2015)

13. J. Raven, Cognitive Psychology, 41, 1 (2000)

14. Y.V. Kovas, T.N. Tikhomirova, S.B. Malykh, Voprosy psihologii, 6, 67 (2011)
15. R. Colom, F.J. Abad, L.F. Garci'a, M. JuanEspinosa, Intelligence, 30, 449 (2002)

16. B. Oliver, N. Harlaar, M. Hayiou-Thomans, Y. Kovas, S. Walker, F. Spinath, S. Petrill, P. Dale and R. Plomin, Journal of Educational Psychology, 96, 504 (2004)

17. S. von Stumm and R. Plomin, Intelligence, 48, 30 (2015)

18. E.M. Tucker-Drob and D.A. Briley, Psychological Bulletin, 140, 949 (2014)

19. S.A. Brouwers, F.J.R. van de Vijver, D.A. van Hemert, Learning and Individual Differences, 19, 330 (2009)

20. M.W. Watkins, P.W. Lei and G.L. Canivez, Intelligence, 35, 59 (2007). 